\title{
Low-frequency Alfvén eigenmodes during the sawtooth cycle at ASDEX Upgrade
}

\author{
D Curran ${ }^{1}$, Ph Lauber ${ }^{2}$, P J Mc Carthy ${ }^{1}$, S da Graça ${ }^{3}$, V Igochine ${ }^{2}$ and \\ the ASDEX Upgrade Team ${ }^{2}$ \\ ${ }^{1}$ Department of Physics, University College Cork, Association EURATOM-DCU, Cork, Ireland \\ ${ }^{2}$ Max-Planck-Institut für Plasmaphysik, EURATOM-Association IPP, Garching, Germany \\ ${ }^{3}$ Associação EURATOM/IST, Instituto de Plasmas e Fusão Nuclear - Laboratório Associado, \\ Insituto Superior Técnico, 1049-001 Lisboa, Portugal \\ E-mail: d.b.curran@umail.ucc.ie
}

\begin{abstract}
.
The confinement of fast particles, present in tokamak plasmas as nuclear fusion products and through external heating, will be essential for any future reactor. Fast particles can be expelled from the plasma through their interaction with Alfvén eigenmode (AE) instabilities. AEs can exist in gaps in the Alfvén continuum created by plasma equilibrium non-uniformities. In ASDEX Upgrade low frequency modes in the Alfvén-acoustic frequency regime, including betainduced Alfvén eigenmodes (BAEs) and lower frequency modes with mixed Alfvén and acoustic polarizations, have been observed. They exist in gaps in the Alfvén continuum opened up by geodesic curvature and finite plasma compressibility. In this paper a kinetic dispersion relation (Lauber Ph et al 2009 Plasma Phys. Control. Fusion 51 124009) is solved numerically to investigate the influence of diamagnetic effects on the evolution of these low frequency modes during the sawtooth cycle. Other distinct but potentially related modes which sweep significantly upwards in frequency towards the end of the sawtooth cycle are also considered. Using information gained from soft x-ray measurements (Igochine V et al 2010 IPP Report 1/338) and electron temperature information from electron cyclotron emission to constrain the safety factor profiles, realistic equilibrium reconstructions for the analysis are obtained using the CLISTE code (Mc Carthy P J 2012 Plasma Phys. Control. Fusion 54 015010). The results for the mode frequency evolution are then compared with experimental results from ASDEX Upgrade.
\end{abstract}




\section{Background}

\subsection{Theory}

Shear Alfvén waves are transverse low frequency electromagnetic waves which satisfy the dispersion relation $\omega=k_{\|} v_{A}$, where $k_{\|}$is the wave number in the direction of the magnetic field and $v_{A}=B / \sqrt{\mu_{0} \rho}$ is the Alfvén speed [1]. In the case of a uniform plasma with no kinetic effects included, shear Alfvén waves are dispersionless due to the fact that the phase velocity $\omega / k_{\|} \mathbf{b}$ and group velocity $\partial \omega / \partial \mathbf{k}$ are equal. However, in the case of an ambient magnetic field with an azimuthal component, periodicity constraints require that $k_{\|}=(n-m / q) / R_{0}$ [1]. This becomes dependent on the radial location through a non-uniform safety factor profile $q(r)$, whose radial dependence is in turn due to the form of $B$ from Ampére's law. Waves that obey $\omega(r)=k_{\|}(r) v_{A}$ form part of the shear Alfvén continuum that extends across the plasma minor radius, and wave packets propagating in the plasma are quickly dispersed due to phase mixing caused by adjacent waves having different phase velocities. Thus, most propagating waves are rapidly damped at a rate that is proportional to the gradient of the phase velocity [1]. An overview of the basic physics of the shear Alfvén continuum can be found in [1]. The idea of plasma heating by shear Alfvén waves via phase mixing was first investigated in [2]. If we now consider a toroidal plasma, various geometric effects can act to open gaps in the shear Alfvén continuum and discrete modes can exist within these gaps. The resultant MHD mode spectra have been investigated in $[3,4,5]$. It has been found that these discrete modes can in turn be excited through interactions with charged fusion products and fast ions caused by external heating [6], as well as gradients in the background plasma profiles [7]. The main classes of continuum gap formed are those caused by the intersection of the dispersion curves of counter propagating waves [8] and those formed by the existence of an extremum in the shear Alfvén continuum $[9,10,11]$. A well known example of the former is the toroidicityinduced Alfvén eigenmode (TAE) which can exist in the gap opened up due to poloidal symmetry breaking [8]. In this work we will focus on the low-frequency gap that can form beneath the Alfvén continuum known as the $\beta$-induced gap, first studied in detail in [11]. This gap is caused by the compressional response of the plasma to shear Alfvén waves in the presence of finite pressure and geodesic curvature [11, 12, 13]. Betainduced Alfvén eigenmodes (BAEs), are electromagnetic, $n \neq 0$ perturbations, with a polarization close to that of shear Alfvén waves $[12,14]$. They appear to be driven by strong gradients in temperature and density as well as by fast particle popluations, and exist within the $\beta$-induced gap $[11,14,15]$. Once these gaps are formed, the degeneracy of the eigenmodes with the continuum accumulation point, which is the point where the continuum has a local extremum, can be removed and the modes become weakly damped $[13,16]$. This allows modes to potentially be driven unstable by resonant wave-particle interactions $[7,13]$. Thus, the various characteristic frequencies relevant in the lowfrequency gap become important $[7,17]$. For example, the well circulating ion resonance condition is dependant on the transit frequency, while particles trapped as a result of a 
magnetic well have a resonance condition which depends on the bounce and toroidal drift precession frequencies $[7,13,17]$. As suggested in the abstract, the interaction between fast particles and these Alfvén eigenmode instabilities can have a significant effect on their confinement [18]. Whereas BAEs have a mainly Alfvénic polarization, another type of mode can exist in the $\beta$-induced gap that has mixed Alfvénic and acoustic polarization in the radial region surrounding the continuum accumulation point and with a frequency lower than that of the $\operatorname{BAE}[7,13,16,18,19,20]$. They appear in the same frequency regime as the beta-induced acoustic Alfvén eigenmode (BAAE), which has been investigated in the ideal MHD [18] and kinetic [20] framework, and will be referred to as "low-frequency modes" in this work. Considerably increased fast particles losses due to coupling between BAEs and TAEs have already been well established $[7,12]$. Thus, an understanding of this lower frequency branch in the BAAE frequency range, which could possibly enhance these fast particle losses through coupling to the higher frequency modes, is potentially very important.

\subsection{Experiment}

Low frequency modes have been observed in the frequency range between that of the kink instability and TAE, which are typically observed in ASDEX Upgrade at $\leq 10 \mathrm{kHz}$ and $\geq 150 \mathrm{kHz}$ [21] respectively. These modes are localized in the region surrounding the $q=1$ surface and have been observed during ICRH [14], ECRH and NBI [22] heated discharges. We provide evidence below to indicate that the modes which are visible in the frequency range $f \approx 60-75 \mathrm{kHz}$ in figure 1 are BAEs, while the low frequency modes observed in the BAAE frequency regime between $f \approx 10$ and $45 \mathrm{kHz}$ are of a mixed Alfvénic and acoustic polarization. In addition to these, modes which sweep upwards in frequency to nearly twice that of the maximum BAE frequency towards the end of the sawtooth cycle have also been observed in certain ASDEX Upgrade discharges. The term sweeping is used so as to emphasize the medium time-scales, on the order of tens of milliseconds, over which these modes are observed to evolve. These three types of modes will be investigated in this work.

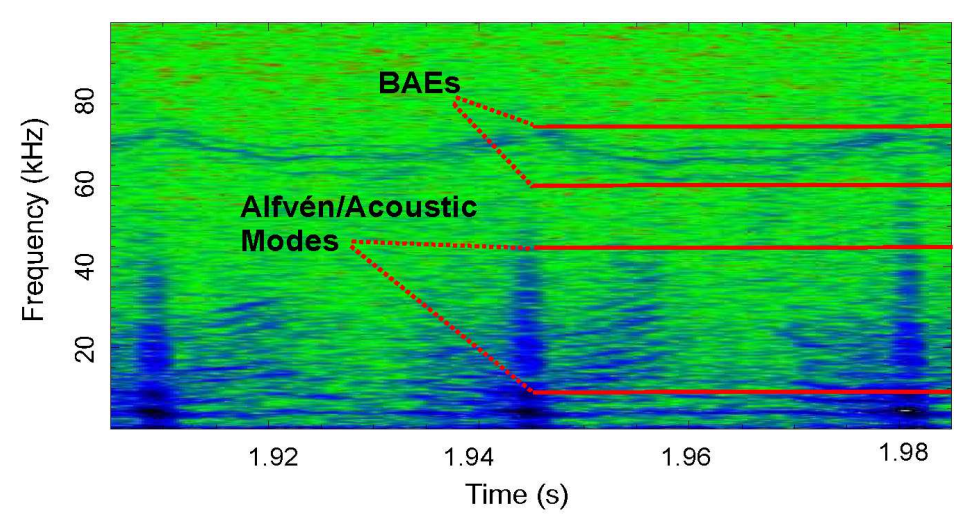

Figure 1. Low frequency mode activity during two sawtooth cycles from $t=1.91-1.98$ s for discharge 25546 measured with core $\left(\rho_{\text {pol }} \approx 0.24\right)$ soft x-ray channel I51.
While the BAEs are clearly observed from edge magnetic field perturbations using Mirnov coils, the low-frequency modes are not. Using soft x-ray (SXR) measurements in the core, both the BAEs and the low-frequency modes are observed clearly (figure 1). It can be inferred from these observations that the BAEs have a mainly Alfvénic polarization which causes a large 
enough magnetic field perturbation so as to be observed at the plasma edge. The low-frequency modes are taken to have a lower proportion of Alfvénic polarization to the BAEs based on the fact that they are not visible from edge magnetic measurements. However, they are observed to have amplitudes comparable to the BAEs in the core region in the BAAE frequency range. They are also found to have similar mode numbers and radial localization to the BAEs [7], suggesting that they are related in character to the BAEs. They are observed in a frequency regime that suggests that they are related to coupling to KBMs through the $\omega_{* p}$ branch [14][16], where $\omega_{* p}$ is the diamagnetic frequency resulting from gradients in the background temperature and density profiles. This dependence on $\omega_{* p}$ is observed clearly in the numerical results presented in section 3.2 which agree well with experimantal observations. Experiments are planned to substantiate these conclusions with measurements of $\delta T_{e}$ and $\delta n_{e}$ using improved 2-D electron cyclotron emission imaging (ECEI) and reflectometry diagnostics, respectively, at ASDEX Upgrade to clearly identify the polarizations of these modes. The relative Alfvénic and acoustic contributions to the polarizations of the BAEs and low-frequency modes has also been verified via global eigenvalue solutions of the underlying system of equations [7]. A third branch known as the electrostatic drift wave branch, which has been studied in [23], is recovered numerically but is so heavily damped that it does not appear experimentally. This will be investigated in section 3.2.

\section{Beta-induced Alfvén eigenmodes}

\subsection{Experimental observations of BAEs}

As mentioned in section 1.1, BAEs occur in the low-frequency $\beta$-induced gap beneath the shear Alfvén continuum opened up by geodesic curvature and finite ion compressibility $[12$, 14]. Unlike TAEs, where the gap is formed by the crossing of counter propagating waves with adjacent poloidal harmonics, BAEs exist beneath an extremum in the Alfvén continuum known as the frequency accumulation point. This forms close to the $q=1$ surface where $k_{\|} \approx 0$. Discharge 25546 exhibits mode activity characteristic of BAEs and is a medium density discharge with $n_{e 0} \approx$ $4.75 \times 10^{19} \mathrm{~m}^{-3}$ during the times considered and $I_{p} \approx 800 \mathrm{kA}$. During the time-period $t=1.945-1.979 \mathrm{~s}$, which exhibits sawtooth activity, 4.395MW of ICRH is employed. Lowfrequency modes are observed close to the $q=$

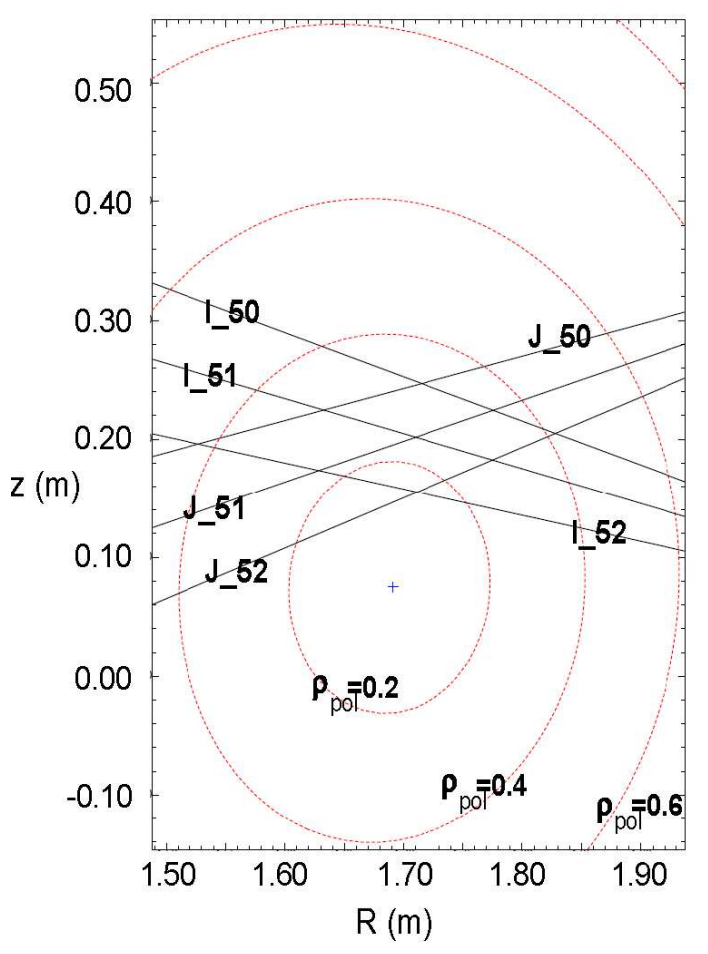

Figure 2. Lines of sight of core channels of soft x-ray cameras I and J, and core flux surface geometry for discharge 25546 at $t=1.955 \mathrm{~s}$. 1 surface from SXR [24] measurements with a tangency radius of $\rho_{\text {pol }} \approx 0.24$ and also 
from magnetics measurements. Mode frequency splitting is evident and the various splittings are estimated, using magnetics measurements from Mirnov coils, to correspond to toroidal mode numbers $n=3,4,5$ and 6 in order of decreasing frequency. According to figure 1 , the frequencies of the different mode splittings dip by several $\mathrm{kHz}$ during the early sawtooth period before plateauing for up to $10 \mathrm{~ms}$ and recovering to their initial value in the final third of the cycle. This behaviour is observed to be related to the temperature gradient evolution during the sawtooth cycle and this will be investigated more closely in section 2.3 .

\subsection{Analysis procedure}

In order to investigate this behaviour a kinetic dispersion relation, which has been re-derived for the gyrokinetic model underlying the eigenvalue code LIGKA [14, 16], was solved numerically. This is derived from the system of quasineutrality, gyrokinetic and gyrokinetic moment (GKM) equations, with only the coefficients of the second order radial derivatives of the GKM considered, the details of which can be found in $[14,16]$. Specific kinetic effects appear in this analysis as, due to the inclusion of finite core ion compressibility, wave-particle resonances can now occur for modes with $\omega \approx \omega_{t i} \approx \omega_{* p}[14,16] . \omega_{t i}$ is the ion transit frequency, defined after equation (1). Diamagnetic effects become important when finite temperature gradients are present. The term 'kinetic dispersion relation' is used in this work to distinguish it from the purely MHD dispersion relation, as presented in [18]. However, the MHD limit can also be recovered analytically from the system of equations mentioned above $[14,16]$. The kinetic dispersion relation is comprised of polynomials in $x_{m \pm 1}=\omega / \omega_{t i}$ and $Z\left(x_{m \pm 1}\right)$, defined in equations (1) and (2), where $x_{m \pm 1}$ is a measure of the degree of resonance between the modes and passing particles, and where $Z\left(x_{m \pm 1}\right)$ is the plasma dispersion function. Thus, specific kinetic effects enter into the kinetic dispersion relation and alter the value of the calculated continuum frequency and drive/damping, as well as allowing one to maintain the vanishing highest derivative terms in the system as in the ideal MHD case. In the following paper, when the term 'continuum' is used, it will refer to the continuum calculated using this kinetic dispersion relation as derived via the kinetic analysis in $[14,16]$. Keeping the $m \pm 1$ sidebands, retaining the geodesic curvature and the sound wave coupling by an appropriate approximation of the propagator integrals [14], leads to:

$$
\begin{aligned}
& \omega^{2}\left(1-\frac{\omega_{* p}}{\omega}\right)-k_{\| m}^{2} R_{0}^{2} \frac{\omega_{A}^{2}}{\omega_{A 0}^{2}}=2 \sqrt{\frac{2}{1+\kappa^{2}}} \frac{v_{t h i}^{2}}{R_{0}^{2} \omega_{A 0}^{2}}\left(-\left[H\left(x_{m-1}\right)+H\left(x_{m+1}\right)\right]\right. \\
&\left.+\tau\left[\frac{N^{m}\left(x_{m-1}\right) N^{m-1}\left(x_{m-1}\right)}{D\left(x_{m-1}\right)}+\frac{N^{m}\left(x_{m+1}\right) N^{m+1}\left(x_{m+1}\right)}{D\left(x_{m+1}\right)}\right]\right)
\end{aligned}
$$

where $x_{m}=\frac{\omega}{k_{\| m} v_{t h}}, v_{t h i}^{2}=\frac{2 T_{i}}{m_{i}}, \omega_{t i}=v_{t h i} / q R_{0}, \tau=T_{e} / T_{i}, \omega_{* p_{s}}=\omega_{* n_{s}}+\omega_{* T_{s}}=$ $\frac{T_{s}}{e B} k_{\theta}\left(\frac{\nabla n_{s}}{n_{s}}\right)(1+\eta)$ with $\eta=\frac{\nabla T_{s}}{T_{s}} / \frac{\nabla n_{s}}{n_{s}}, s$ is the species index, and $D, H$ and $N$ represent the sound wave, geodesic curvature and coupling terms respectively with, 


$$
\begin{aligned}
& D\left(x_{m}\right)=\left[1+\tilde{D}\left(x_{e, m}\right)\right]+\tau\left[1+\tilde{D}\left(x_{i, m}\right)\right] \\
& H\left(x_{m}\right)=\tilde{H}\left(x_{i, m}\right)+\tau \tilde{H}\left(x_{e, m}\right) \\
& N^{m}\left(x_{m}\right)=\tilde{N}^{m}\left(x_{i, m}\right)-\tilde{N}^{m}\left(x_{e, m}\right)
\end{aligned}
$$

and,

$$
\begin{aligned}
\tilde{D}(x) & =\left(1-\frac{\omega_{* p}}{\omega}\right) x Z(x)-\frac{\omega_{* p}}{\omega} \eta\left(x^{2}+x Z(x)\left(x^{2}-\frac{1}{2}\right)\right) \\
\tilde{H}(x) & =\frac{1}{2}\left[\left(1-\frac{\omega_{* p}}{\omega}\right) \tilde{F}(x)-\eta \frac{\omega_{* p}}{\omega} \tilde{G}(x)\right] \\
\tilde{F}(x) & =\frac{1}{2}\left(x Z(x)\left(\frac{1}{2}+x^{2}+x^{4}\right)+\frac{3 x^{2}}{2}+x^{4}\right) \\
\tilde{G}(x) & =\frac{1}{2}\left(x Z(x)\left(\frac{3}{4}+x^{2}+\frac{x^{4}}{2}+x^{6}\right)+2 x^{2}+x^{4}+x^{6}\right) \\
\tilde{N}^{m}(x) & =\frac{1}{2}\left(\left(1-\frac{\omega_{* p}^{m}}{\omega}\right)\left[x^{2}+x Z(x)\left(x^{2}+\frac{1}{2}\right)\right]-\frac{\omega_{* p}^{m}}{\omega} \eta\left[\left(x^{4}+\frac{x^{2}}{2}\right)+x Z(x)\left(\frac{1}{4}+x^{4}\right)\right]\right)(3)
\end{aligned}
$$

$Z(x)$ the plasma dispersion function and in equation $(3) \omega_{* p}$ is the diamagnetic frequency for the respective species [14]. Equation (1) has been modified in order to take account of plasma elongation effects [25]. Equilibrium reconstructions were generated using the CLISTE code $[26,27]$ in order to obtain fitted q-profiles. An estimate for the minimum on-axis safety factor value $q_{0}$ was obtained by a non-linear regression using the above dispersion relation, with $q_{0}$ as a parameter and the mode frequency and mode numbers as data. During the course of the sawtooth cycle, this was assumed to fall from $q_{0}<1.00$ to $q_{0} \approx 0.96$, the lower value being recovered through CLISTE honouring the $q=1$ radius estimate from ECE measurements. The $q=1$ radius was assumed to coincide with the sawtooth inversion radius which, for reasons discussed in section 2.3, is taken approximately to be at a fixed radius throughout the sawtooth cycle. Thus, the $q=1$ surface was taken to remain at a fixed radius for the duration of the sawtooth cycle, which is in agreement with [28]. This information was used to constrain the qprofiles. The plasma elongation was determined to be approximately $\kappa=1.25$ in the region of interest and an estimation of the ion density profile was obtained through the availability of $Z_{\text {eff }}$ profiles [29]. A reliable determination of the plasma toroidal rotation frequency for discharge 25546 was not possible so a conservative estimate of $f_{\text {rot }}=1.5 \mathrm{kHz}$ was used in the calculations.

\subsection{Background profile evolution for discharge 25546}

It has been demonstrated that the background temperature profiles play an important role in determining the BAE frequency evolution [7, 13]. During a sawtooth cycle, the temperature is subject to rapid and often drastic variations in the core region of the plasma. A sawtooth period consists of a rapid increase in the core temperature, which saturates in the final stages of the period before relaxing to a significantly lower 
value after the crash. It is brought about by the occurence of a $(1,1)$ kink mode in the plasma, implying the existence of a $q=1$ surface. Throughout the period of sawtooth activity, there is a radial location where the electron temperature remains approximately constant from one sawtooth cycle to the next, which is known as the sawtooth inversion radius [30]. The $q=1$ surface is known to exist somewhere in the region of the sawtooth inversion radius. However, its exact location is dependant on the safety factor and temperature profiles before the crash, as well as on the model used to explain the sawtooth process. Upon assuming parabolic $q$ and $T_{e}$ profiles as well as the Kadomtsev model, the $q=1$ surface is found to coincide with the sawtooth inversion radius [31]. This will not be the case for an extremely flat core safety factor profile with rapid variation outside the $q=1$ surface [31]. Without detailed computer simulations, the assumption that the radial location of the $q=1$ surface and sawtooth inversion radius coincide is the best that can be achieved, and is adequate for this analysis.

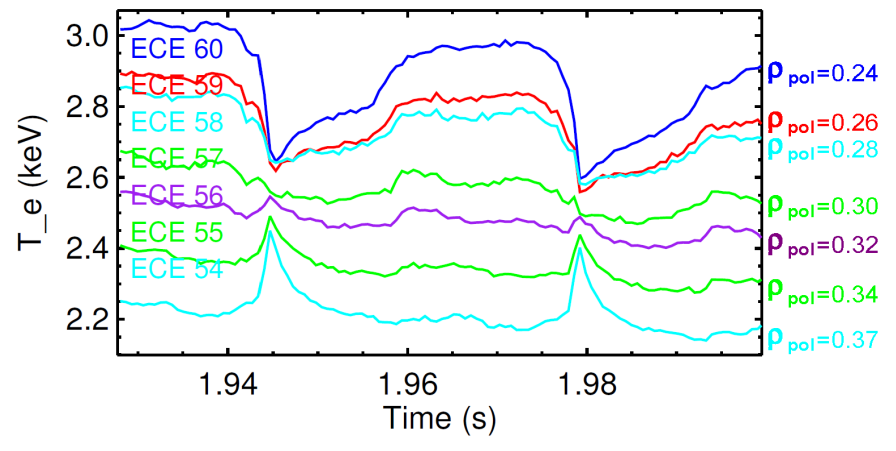

(a)

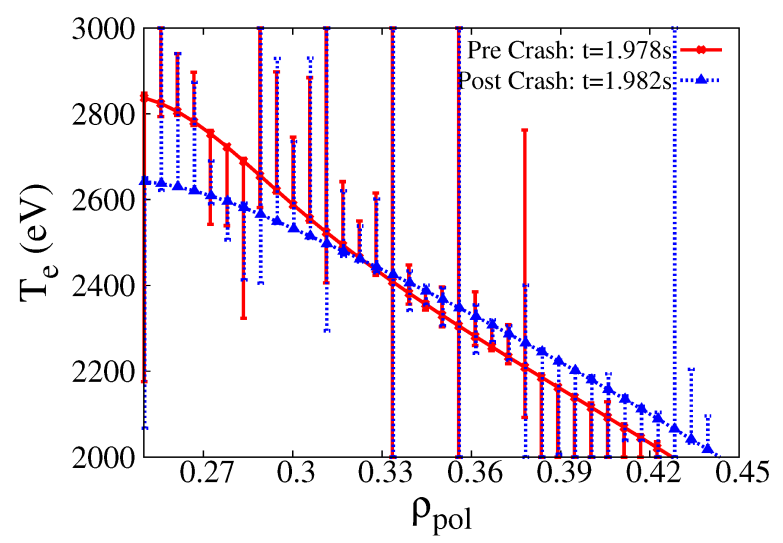

(b)

Figure 3. (a) Time-traces of core ECE channels during sawtooth cycle $t=1.945-1.979 \mathrm{~s}$ (b) Electron temperature profiles from integrated data analysis (IDA), just before $(t=1.978 \mathrm{~s})$ and after $(t=1.982 \mathrm{~s})$ sawtooth crash at $t=1.980 \mathrm{~s}$.

are large where the distance between neighbouring ECE channels is larger than the correlation length used in the $\chi^{2}$-binning method [32]. Thus, large variations in the uncertainty in $T_{e}$ can exist between adjacent radial locations. Figure 3 (b) shows that of core $\left(\rho_{\text {pol }} \approx 0.24-0.37\right)$ ECE channels during a sawtooth cycle from $t=1.945-1.979 \mathrm{~s}$, as well as partial periods of the preceeding and subsequent cycles. The electron temperature read from ECE channel 56, which is at $\rho_{\text {pol }} \approx 0.32$, appears to exhibit the least variation over the period before and after the sawtooth crash at $t=1.980 \mathrm{~s}$. From this it can be inferred that the $q=1$ surface occurs at approximately this radial location. This conclusion is supported by considering the radial location of the intersection of the $T_{e}$ profiles (sawtooth inversion radius), obtained from integrated data analysis of the ECE and Thomson scattering diagnostics (IDA) [32], before and after the crash at $t=1.980 \mathrm{~s}$. Temperature profiles calculated using IDA are determined using ECE data and error bars are estimated using a $\chi^{2}$ binning method [32]. Uncertainties 
the sawtooth inversion radius occurs at $\rho_{\text {pol }} \approx 0.32$. It is observed that the evolution of the BAE mode frequency appears to be influenced by the behaviour of the electron temperature gradient close to the $q=1$ surface. Figure 4 shows BAE mode activity measured using SXR channel J51 with a tangency radius of $\rho_{\text {pol }} \approx 0.22$. BAE mode activity is also visible with the adjacent channel J50 which has a tangency radius of $\rho_{\text {pol }} \approx 0.30$, suggesting that the BAEs are reasonably radially extended. Figure 4 also shows the difference in $T_{e}$ measured by neighbouring ECE channels close to the $q=1$ surface. This gives an illustration of the local electron temperature gradient as the radial locations of the ECE measurements can reasonably be assumed fixed in space over the duration of a single sawtooth period.

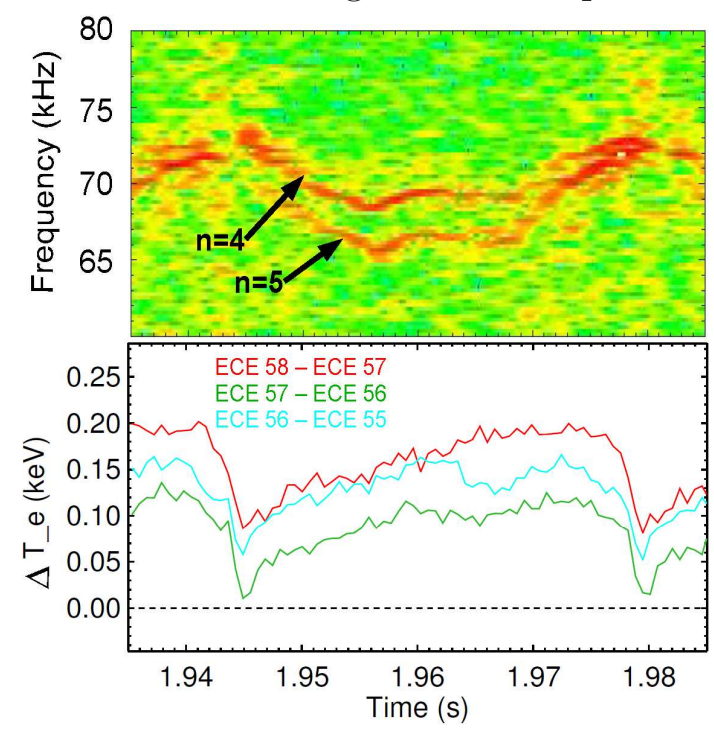

(a)

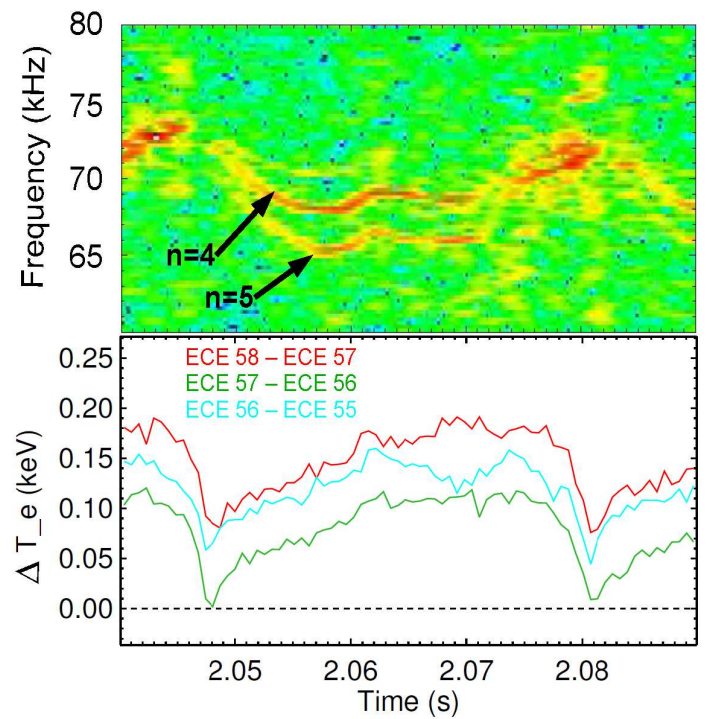

(b)

Figure 4. BAE mode activity measured with SXR channel J51 with tangency radius of $\rho_{\text {pol }} \approx 0.22$ and corresponding difference in $T_{e}$ measured by neighbouring ECE channels close to the $q=1$ surface during two sawtooth periods of discharge 25546 for (a) $t=1.945-1.980 \mathrm{~s}$ and (b) $t=2.048-2.080 \mathrm{~s}$ (ECE channels $58,57,56 \& 55$ are at $\rho_{\text {pol }} \approx 0.28,0.30,0.32$ and 0.34 respectively).

As presented in figure 4, the difference in $T_{e}$ between ECE channels 56 and 57 reduces to approximately zero, within experimental error, at the occurence of the sawtooth crashes, suggesting that the $T_{e}$ profile flattens significantly here. The observed electron temperature gradient behaviour is used to give a further indication as to the approximate location of the $q=1$ radius, based on the knowledge of the approximate sawtooth inversion radius from theory [30] and experiment and the fact that this $\nabla T_{e}$ behaviour at the crash is repeatedly observed over the course of many sawtooth periods. A definite correlation can be seen between the BAE frequency and estimated $\nabla T_{e}$ close to the $q=1$ surface (green time-traces in figure 4). Considering the first time-slice, for $t=1.945-1.957 \mathrm{~s}$ the frequency can be seen to dip by up to $5 \mathrm{kHz}$ for both mode splittings ( $n=4 \& n=5$ from magnetics). During the same period, the gradient close to the $q=1$ surface steepens. From $t=1.959 \mathrm{~s}$, the gradient experiences a moderate 
fall off from its peak value and remains quite constant from $t=1.959-1.969 \mathrm{~s}$. The frequency appears to follow the gradient evolution as it remains approximately constant for these $10 \mathrm{~ms}$. At $t \approx 1.970$ s the local gradient begins to decrease and the mode frequency is seen to sweep back up to its value at the beginning of the cycle. Similar behaviour is observed for the time-slice $t=2.048-2.080 \mathrm{~s}$.

\subsection{Numerical results for BAEs}
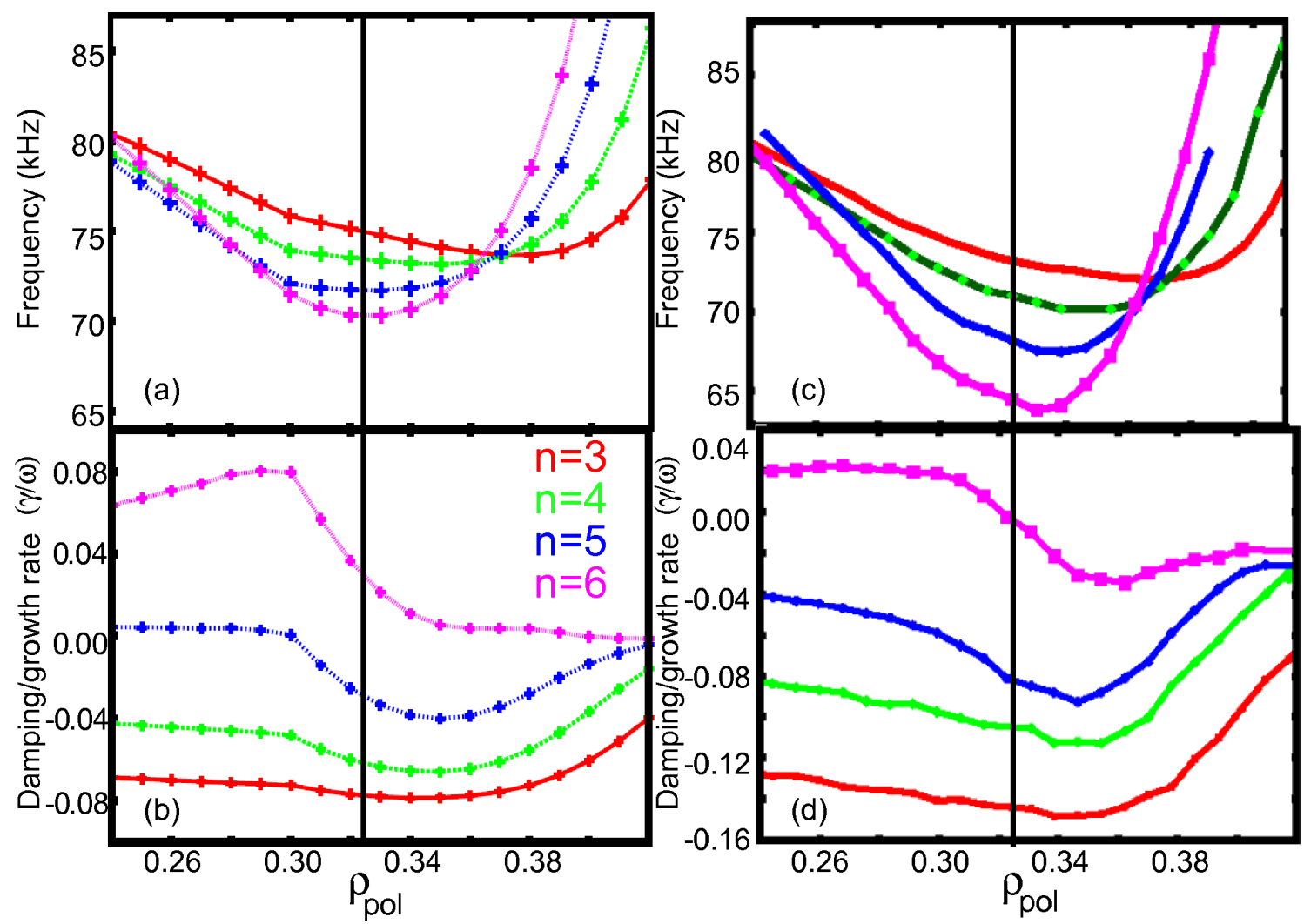

Figure 5. (a) Real frequency continuum and (b) damping/growth rates $(\gamma / \omega)$ for toroidal mode numbers $n=3-6$, determined by solution of analytically derived kinetic dispersion relation, without trapped particle mode frequency splitting effects. (c) Real frequency continuum and (d) damping/growth rates $(\gamma / \omega)$ for toroidal mode numbers $n=3-6$, determined by full numerical calculation using LIGKA with trapped particle effects fully taken into account. The rational surface at $\rho_{\text {pol }} \approx 0.32$ is indicated by vertical black lines.

The diamagnetic frequency $\omega_{* p}$ is proposed to play an important role in determining the BAE frequency and its contribution is taken into account by its presence in the kinetic dispersion relation $[14,16]$. Due to the fact that $\omega_{* p} \propto n \nabla T$ (In this case $T_{i}=T_{e}$ is assumed), the effects of variations with $\nabla T_{e}$ during the sawtooth cycle, as well as with different toroidal mode numbers $n$, were investigated. As BAEs are observed to be localized in the region surrounding the $q=1$ surface, the focus was on the evolution of the $T_{e}$ profile in this region so as to ascertain its effects on the BAE frequency 
accumulation point. It has been demonstrated in [17] that the interaction of particles trapped within magnetic wells with low frequency modes becomes important for mode frequencies of the order of the ion bounce $\omega_{B i}$ and drift precession $\omega_{D i}$ frequencies. Figure 5 compares two calculations of the real frequency continuum and growth/damping rates for toroidal mode numbers $n=3-6$ at $t=1.955$ s for discharge 25546 . The first was determined by a full numerical solution of the system of gyrokinetic moment, quasineutrality and gyrokinetic equations underlying the linear eigenvalue code LIGKA [14], with trapped particle effects fully included. The second was a numerically calculated solution of the analytically derived kinetic dispersion relation, without trapped particle mode frequency splitting effects. In both cases, a $1.5 \mathrm{kHz}$ toroidal plasma rotation frequency has been included in the results for the real continuum. Due to the fact that the effects of trapped particle dynamics have not been included in the analytical expression, a significant difference in the quantitative results was observed between this and the full numerical solution obtained by LIGKA. From calculations with LIGKA, when trapped particle effects are considered in addition to the standard circulating particle case, a frequency downshift of approximately $25 \%$ is observed.

Thus, a 25\% additional downshift has been assumed in the analytically calulated mode frequency due to the effects of trapped particle dynamics on low frequency modes. This assumption has been verified by full numerical calculations of the frequency continuum $[7,17]$. The radial locations of the BAE accumulation points for the different mode numbers agree closely for both cases, with the higher $n$ modes localized closer to the $q=1$ surface. This suggests that diamagnetic effects play an important role in localizing the BAE frequency accumulation point to the region close to the $q=1$ surface, as $\omega_{* p} \propto n \nabla T$.

The dependence of the continuum accumulation point frequency on the radial coordinate, in this case the normalized poloidal radius $\rho_{\text {pol }}$, can be understood by considering how the inclusion of finite- $\beta$ effects alter the continuous spectrum. In the case of this analysis, an estimate for the $q=1$ radius of $\rho_{\text {pol }} \approx 0.32$ was determined. If one considers a purely shear Alfvén wave with dispersion relation $\omega=k_{\|} v_{A}$, with $k_{\|}=(n-m / q) / R_{0}$, there will be no low frequency gap and hence no BAE continuum accumluation point. $\omega=0$ will occur at the $q=1$ radius for all modes with equal toroidal and poloidal mode numbers $(n=m)$ due to the form of $k_{\|}$. If we now include the effects of finite core ion compressibility and geodesic curvature as presented in $[14,16]$, then the low frequency $\beta$-induced gap is opened and the BAE continuum takes a form determined by solving equation (1) for each radial point. This equation contains different powers of $\omega_{* p}$ which are proportional to the toroidal mode number $n$ and have a radial dependence from $T\left(\rho_{p o l}\right), \partial T\left(\rho_{p o l}\right) / \partial \rho_{p o l}, n\left(\rho_{p o l}\right), \partial n\left(\rho_{p o l}\right) / \partial \rho_{p o l}$ and $B\left(\rho_{\text {pol }}\right)$. If we take the derivative of this equation with respect to the radial coordinate $\rho_{\text {pol }}, \partial \omega / \partial \rho_{\text {pol }}=0$ will be no longer occur simply where $k_{\|}=0$ as a result of these additional terms, defined following equation (1), and the BAE continuum accumulation point will be shifted radially somewhat by an amount dependant on the mode toroidal mode numbers. 
The differences in the calculated mode frequencies at the continuum accumulation points between figures 5 (a) and (c) for modes $n=4-6$ range from $3-6 \mathrm{kHz}$. Thus, it is clear that to fully model the mode splitting caused by the fact that $\omega_{* p} \propto n \nabla T$, the inclusion of the trapped particle terms in the analytical expression is required for recovering the correct low frequency mode frequencies $[17,7]$. This is due to the fact that the trapped particle terms are also dependant on $\omega_{* p}$, and as such, the mode frequency splitting will be altered by them [17]. This is supported by the fact that for the $n=3$ mode, which experiences the smallest $\omega_{* p}$ contribution, the agreement is much better between analytical and full numerical results than for the higher $n$ modes. The discrepancy between the downward frequency shifts can also be partially accounted for by the truncation of higher order terms in the derivation of the analytical expression which are included in the full numerical calculation $[14,16]$.

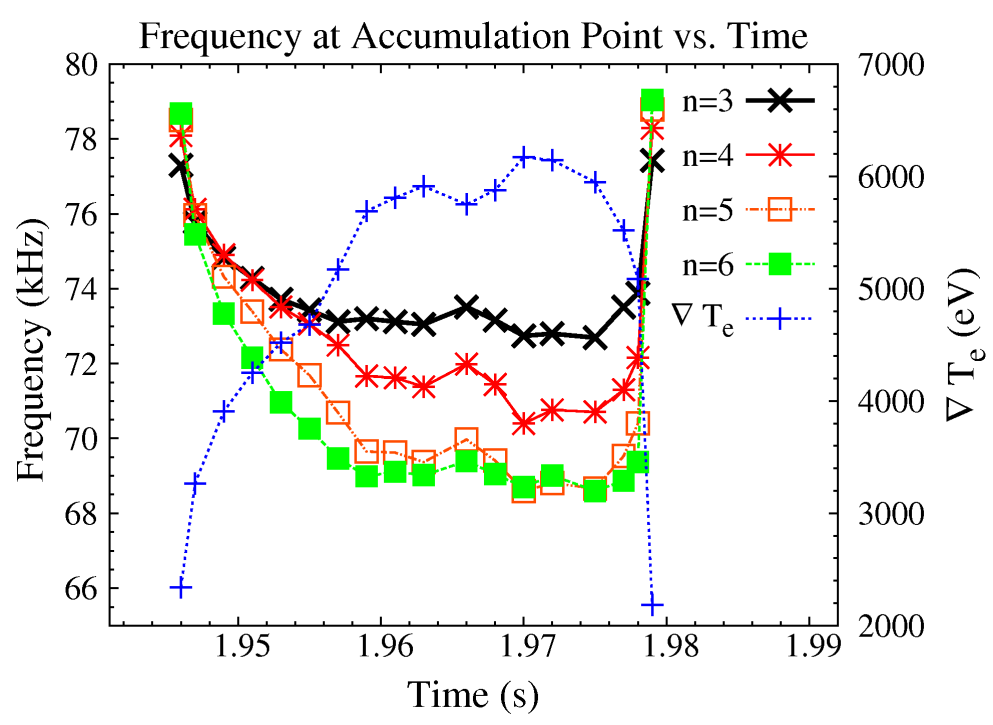

Figure 6. Comparison of calculated mode frequency at accumulation point with changes in the electron temperature gradient $\nabla T_{e}$ at the $q=1$ surface during sawtooth cycle for discharge 25546
Looking at figure 5 (b) and (d), while the qualitative behaviour of the growth and damping rates calculated by LIGKA and by solving the analytic kinetic dispersion agree quite well, they do differ somewhat quantitatively in the region close to the rational surface at $\rho_{\text {pol }} \approx 0.32$. This can again be attributed to the inclusion of trapped particle effects in the LIGKA solutions. For example, the drive for the $n=6$ case, which is the lowest frequency mode considered, is substantially higher in figure 5 (b) than in (c). Due to the fact that Landau damping will increase for lower frequencies, neglecting the trapped particle wave-particle resonances overestimates the drive in this region [7]. In both cases the maximum growth rates or minimum damping rates calculated are relatively high close to the BAE continuum accumulation point. This is due to the high background $T_{e}$ gradients in this region. Results presented in [7] demonstrate that the BAEs are radially localized close to the continuum accumulation point.

Each point plotted in figure 6 represent the frequency of the BAE continuum accumulation point for the toroidal mode numbers $n=3-6$. The actual mode frequency, which can only be recovered by a global analysis, is expected to sit slightly below this frequency [33]. Figure 6 shows the evolution of the BAE continuum accumulation point with the electron temperature gradient $\nabla T_{e}$ (plotted in blue) at the $q=1$ surface during 
a sawtooth cycle from $t=1.945-1.979 \mathrm{~s}$, for discharge 25546 .

The radial location of the $q=1$ surface was assumed to remain fixed at $\rho_{\text {pol }} \approx 0.32$ throughout the cycle, based on experimental observations from ECE and SXR, as well as from theory [28]. The local magnetic shear was found to have a negligible effect on the frequency of the accumulation point. Thus, three safety factor profiles, determined from CLISTE equilibrium reconstructions at $t=1.955 \mathrm{~s}, 1.966 \mathrm{~s} \& 1.973 \mathrm{~s}$, were used in determing the continua in the time regions $t=1.946-1.957 \mathrm{~s}, 1.959-1.966 \mathrm{~s} \&$ $1.968-1.979$ s respectively. Values for $q_{0}$ of $0.979,0.969$ and 0.962 were assumed for the three periods respectively. $\nabla T_{e}$ was obtained from IDA measurements and was observed to increase nearly monotonically during the first $\sim 10 \mathrm{~ms}$ of the cycle. For the next $\sim 10 \mathrm{~ms}$ it assumes a rather constant value before decreasing rapidly in the final stage of the sawtooth period. The degree to which the mode activity depends on $\omega_{* p}$ can be seen by comparing the evolution of $\nabla T_{e}$ and the mode frequencies. For example, at $t=1.951 \mathrm{~s}$, the temperature and density gradient lengths were found to be $\nabla T_{e} / T_{e}=-4.31 \mathrm{~m}^{-1}$ and $\nabla n_{i} / n_{i}=-0.46 \mathrm{~m}^{-1}$ at the $q=1$ surface. From this, with $k_{\theta}=-m / r_{s}, r_{s}=17 \mathrm{~cm}(q=1$ surface location $)$ and $B=2.30 \mathrm{~T}$, the diamagnetic frequency was calculated to be $f_{* p} \approx 19 \mathrm{kHz}$ for the $n=4$ mode, while the BAE mode frequency is $65 \mathrm{kHz}$, after being adjusted for the Doppler shift. At $t=1.957 \mathrm{~s}$, when the experimental BAE frequency reaches a minimum, $f_{* p}$ is calculated to be $23 \mathrm{kHz}$, while the BAE mode frequency is $63 \mathrm{kHz}$. Thus, the diamagnetic frequency is found to be up to $35 \%$ of the BAE frequency in some cases.

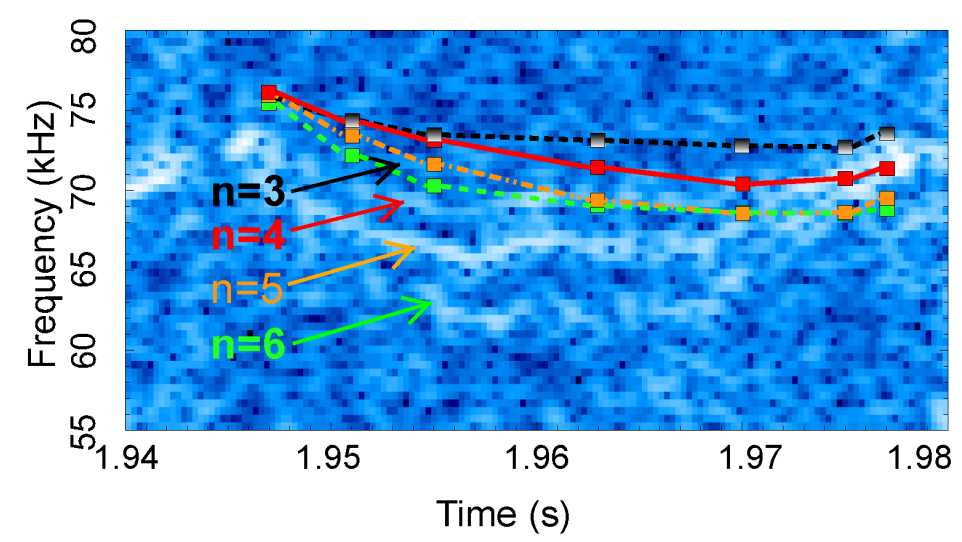

Figure 7. BAE mode activity (diffuse white traces with arrows to identify the mode numbers) measured with core SXR channel I51 $\left(\rho_{\text {pol }} \approx 0.24\right)$ during sawtooth cycle of discharge 25546. Also plotted are the numerically calculated mode frequencies for $n=3-6$.

acter at its highest gradient in time towards the middle of the sawtooth period. As the mode frequency is observed to be directly related to the temperature gradient in this region, this is offered as an explanation for the frequency minimum in time that is reached during this period. A comparison with experimental observations from SXR channel I51, with a tangency radius of $\rho_{\text {pol }} \approx 0.24$, is given in figure 7 . Firstly, consider- 
ing the lower mode numbers $n=3 \& 4$ in figure 7 , good quantitative agreement is found with experiment. The calculated mode frequency is observed to be within $2-3 \mathrm{kHz}$, or $\approx 5 \%$ of the experimentally observed values for the duration of the cycle. The agreement is not as close for the higher mode numbers but is still found to be within $10 \%$ for most time-points considered. Qualitatively, the calculated mode frequency evolution appears to agree well with experiment for the first $\sim 20 \mathrm{~ms}$ of the sawtooth cycle. Comparing figures 6 and 7, the mode frequency evolution appears to follow $\nabla T_{e}$ closely. From figure 7 it is observed experimentally that between $t=1.947 \mathrm{~s}$ and $1.957 \mathrm{~s}$ the $n=4$ and $n=5$ modes experience a $3-4 \mathrm{kHz}$ downshift. This frequency dip is reproduced well by numerical calculations. From $t=1.957-1.970$ s the mode frequencies assume reasonably constant values. This is also reproduced quite well by the numerical results. As an error of $5-10 \%$ is inherent in the experimental value for $\nabla T_{e}$, it was not possible to reproduce reliably the small-scale variations in the mode frequency during this time period. The agreement between experiment and simulation appears to break down to a certain degree in the final $8-10 \mathrm{~ms}$ of the sawtooth cycle. While the mode frequency, observed using SXR, starts to steadily recover to the value it had at the beginning of the cycle , beginning at $t=1.970 \mathrm{~s}$, the numerically calculated frequency does not increase as rapidly. Nevertheless, the calculated BAE frequency for the final time-point, $t=1.979 \mathrm{~s}$, does agree well with the experimentally observed value. Considering the behaviour of $\nabla T_{e}$ during this period, the sawtooth precursor oscillations, which flatten the $T_{e}$ profile close to the $q=1$ surface, do not manifest themselves strongly until the final $4-5 \mathrm{~ms}$ of the cycle. Thus it is clear that other mechanisms are also acting to drive the frequency recovery in the final $\sim 10 \mathrm{~ms}$. One possibility is that a difference in the behaviour of electron and ion temperatures during the precursor phase, and hence in $\nabla T_{e}$ and $\nabla T_{i}$, could be responsible. This effect is excluded in the calculations since $\tau=T_{e} / T_{i}=1$ is assumed but is considered briefly in the section 2.5. Another possibility is the accumulation of impurities towards the end of the cycle. This would act to increase $Z_{\text {eff }}$ and thus drive the calculated mode frequency upwards by increasing the Alfvén speed used in its recovery from the normalized frequency $\omega / \omega_{A 0}$.

\subsection{Influence of $\tau=T_{e} / T_{i}$ on BAE continuum frequency and growth/damping rates}

Ion temperature data was unavailable for discharge 25546. As discharge 25857 had similar heating power and density, ion temperature data from this discharge was used in a parameter scan for discharge 25546. As mentioned in section 2.4, ion and electron temperature profiles could potentially evolve at quite different rates towards the end of the sawtooth cycle. Due to their smaller masses, electrons will respond more quickly to sawtooth precursor oscillations and hence the $T_{e}$ profile should experience a more rapid flattening in the region close to the $q=1$ surface where magnetic reconnection is occuring. This scan was conducted with all other parameters equal to those at $t=1.975 \mathrm{~s}$ in the precursor phase of a sawtooth cycle during discharge 25546. The ion temperature profile was varied between that of the experimental electron and ion temperature profiles 


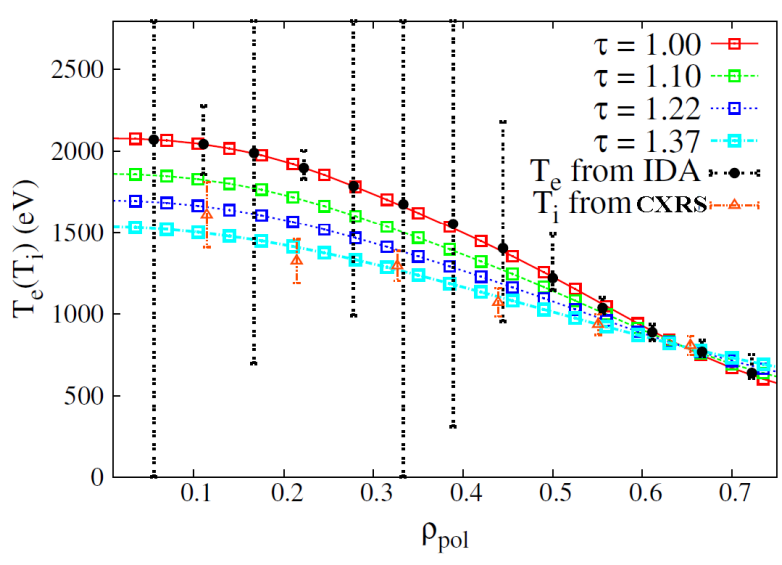

(a)

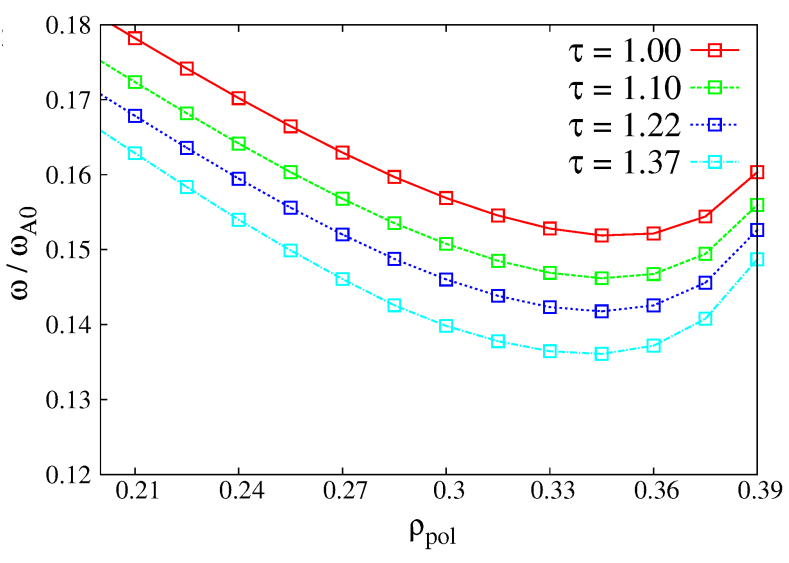

(b)

Figure 8. (a) Core electron and ion temperature profiles for discharge 25857 from IDA and CXRS respectively, as well as model $T_{i}$ profiles. (b) Variation in continuum accumulation point with $\tau$ for $n=4$ mode.

Figure 8 (a) shows the modelled variation in the ion temperature profile, as well as the actual measured electron and ion temperature profiles from IDA and CXRS respectively. $\tau$, which is the ratio between the electron and ion temperature, was varied between the experimental value of $\tau=1.37$ and $\tau=1.00$ in the case where the electrons and ions have the same temperature. Figure 8 (b) shows the effect on the continuum of this variation.

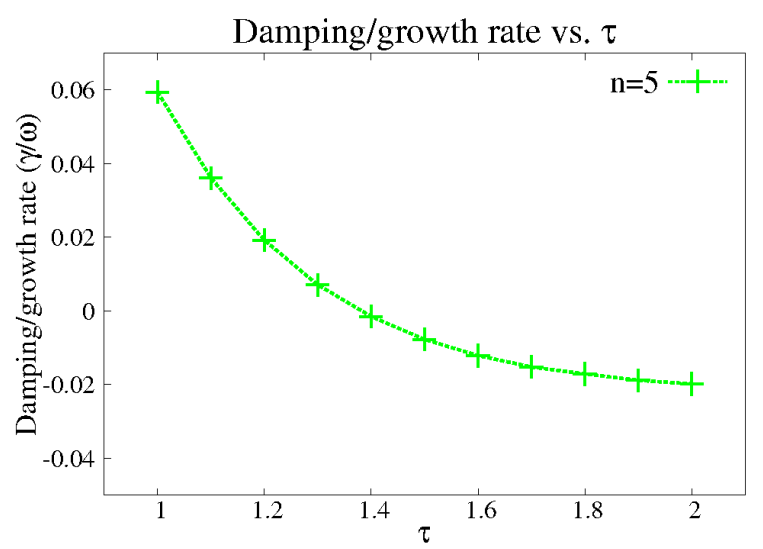

Figure 9. Damping/growth rates $(\gamma / \omega)$ at predicted rational surface $\left(\rho_{p o l} \approx 0.32\right)$ for $n=$ 5 at $t=1.975 \mathrm{~s}$. Note that the modelled $T_{i}$ profiles are scaled down, and hence flattened, with increasing $\tau$, leading to smaller $\nabla T_{i}$ values. This results in reduced mode drive from coupling to the KBM branch, which is provided by $\omega_{* p} \propto$ $\nabla T_{i}$. This compensates the decreased damping from acoustic branch coupling resulting from higher values of $\tau$, leading to an overall decrease in drive.

obtain an accurate result for the BAE mode frequency. As such, its omission in
Decreasing the value of $\tau$ results in an upward shift in the continuum, with the difference in the frequency accumulation points found using the assumed $\tau=1.00$ and the experimentally observed $\tau=1.37$ being up to $10 \%$. During a sawtooth crash, the hot core particles are expelled from the region inside the $q=1$ surface. Thus, it is to be expected that the electron temperature profile should flatten at a faster rate than that of the ions due to the smaller response time of the electrons to this expulsion process. Consequently, $\tau=$ $T_{e} / T_{i}$ will decrease and a more pronounced increase in the BAE frequency in the final stages of the sawtooth cycle would be expected. These simple calculations point to the fact that it is important to take into account the difference in the ion and electron temperature profiles in order to 
the previous calculations may explain to a certain degree the discrepancy between experimental observations and the numerically calculated frequency in the final stages of the sawtooth cycle, when the difference in $T_{e}$ and $T_{i}$ is likely to be greatest.

It is also instructive to consider the effect that a variation in $\tau$ has on the continuum growth/damping rates of the BAE at the predicted mode radial location $\rho_{\text {pol }} \approx 0.32$. As such, a scan over a range of $\tau$ values was conducted, shown in figure 9 , for the $n=5$ continuum at $t=1.975$ s for discharge 25546. A higher value of $\tau$ would be expected to result in reduced damping from acoustic branch coupling. However, in order to model the experiment self consistently the electron temperature, which is well known experimentally from IDA, was kept constant with the ion temperature profiles being scaled down. This results in reduced mode drive from coupling to the KBM branch, which is provided by $\omega_{* p} \propto \nabla T_{i}$. At $\tau=1.0$, the $n=5$ mode experiences reasonable mode drive $(\gamma / \omega \approx 6 \%)$. As the value of $\tau$ is increased, the level of drive decreases, reaching marginal stability at $\tau \approx 1.4$. In this case, the decreased damping from acoustic branch coupling caused by increasing $\tau$ is compensated by the reduced mode drive provided by coupling to the KBM branch. This leads to a reduction in the overall mode drive on balance. A further increase in $\tau$ leads to some additional damping but the rate at which this occurs declines rapidly. Thus, as $\tau$ is increased to predicted experimental values of $\tau \approx 1.4$ the BAE drive is lessened but they nonetheless reach only marginally stability.

\section{Low-frequency modes in BAAE frequency regime}

\subsection{Experimental observations of low-frequency modes in BAAE frequency regime}

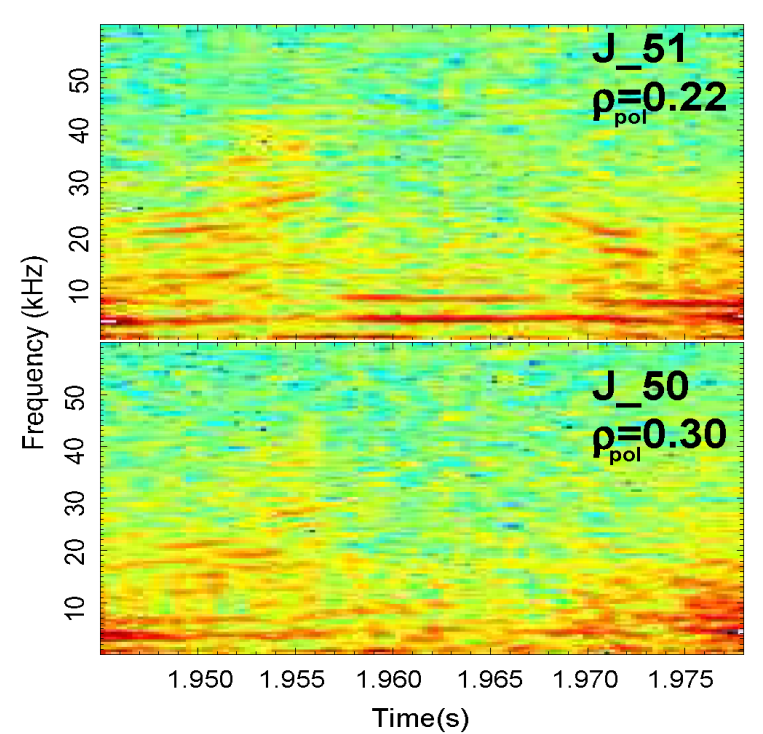

Figure 10. Low frequency mode activity during ICRH $(t=1.945-1.978 \mathrm{~s})$, measured using soft X-ray channels with tangency radii of $\rho_{\text {pol }}=0.22$ and $\rho_{\text {pol }}=0.30$ for discharge 25546 .
As introduced in section 1.2, low-frequency modes of mixed Alfvén and acoustic polarization have been observed in the BAAE frequency regime between $f \approx 10$ and $50 \mathrm{kHz}$ during the sawtooth cycle in certain ASDEX Upgrade discharges. Figure 10 shows an example of these modes, measured by two SXR channels in the core during a sawtooth cycle from $t=1.945-1.979 \mathrm{~s}$, which was heated exclusively by ICRH. These modes are observed most clearly inside the $q=1$ surface, as evidenced by the higher amplitudes measured with the SXR cameras inside $\rho_{\text {pol }} \approx 0.30$. Similarly to BAEs, the frequencies of these modes appear to respond to changes in the temperature gradient during the sawtooth cycle, albeit in 
the opposite manner i.e. they experience an increasing frequency with increasing $\nabla T_{e}$ $(t=1.947-1.955 \mathrm{~s})$ and a frequency reduction with decreasing $\nabla T_{e}(t=1.970-1.977 \mathrm{~s})$. However, they appear to be excited only in the first and last $\sim 10 \mathrm{~ms}$ of the sawtooth period. No clear low-frequency $(10-50 \mathrm{kHz})$ mode activity is visible during the central $\sim 10-15 \mathrm{~ms}$ from $t \approx 1.957-1.968 \mathrm{~s}$. Thus, sufficient mode drive appears absent in this region. It should be noted that the mode activity that is visible under $10 \mathrm{kHz}$ throughout the sawtooth cycle is most likely attributable to the $(1,1)$ kink mode and its harmonics which are not directly related to the low-frequency modes being considered in this analysis.

\subsection{Numerical results for low-frequency modes in BAAE frequency regime}

An example of the real and imaginary continua for a BAE and acoustic/Alfvén mode at $t=1.975 \mathrm{~s}$ is demonstrated in figure 11 . Figure 12 demonstrates results for the low frequency branch recovered by solving the kinetic dispersion relation for a range of mode numbers from $n=2-6$, as well as a comparison with $\nabla T_{e}$ at the approximate $q=1$ surface location from $t=1.946-1.979 \mathrm{~s}$, which is the purely ICRH heated case. It can be seen that the mode frequencies increase early in the sawtooth cycle and decrease towards the end. Also, modes with higher toroidal mode numbers appear at higher frequencies (based on the numerical calculations and opposite to BAEs) and experience a steeper frequency slope in time. This is in qualitative agreement with the experimentally observed behaviour of the lower frequency modes in figures 10 and 13. Quantitatively, the calculated accumulation points are approximately within the correct experimentally observed frequency range

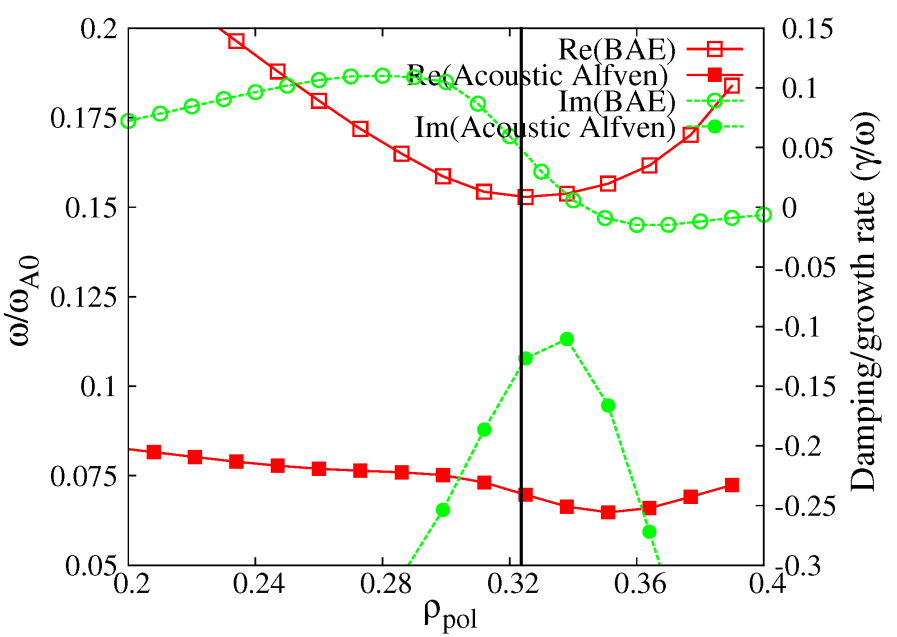

Figure 11. Real and imaginary components of the BAE and low-frequency mode continua for the $n=5$ mode at $t=1.975 \mathrm{~s} . q=1$ rational surface shown at $\rho_{\text {pol }} \approx 0.32$.

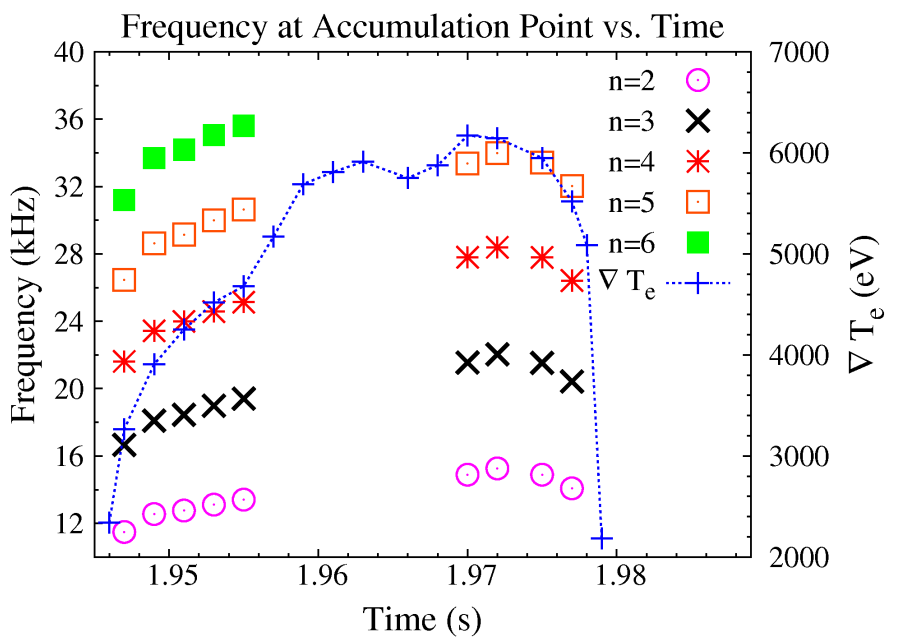

Figure 12. Evolution of low-frequency mode accumulation points for $n=2-6$ and comparison with $\nabla T_{e}$ at the $q=1$ surface for discharge 25546 . (about $70 \%$ of the $n=6$ BAE frequency case, see figures 1 and 7 ). A satisfactory 
explanation has not yet been obtained for the absence of these modes during the middle of the sawtooth period from $t=1.957-1.968 \mathrm{~s}$. This could be attributable to partial magnetic reconnection activity during the sawtooth period removing the mode existence conditions but further investigation is needed. The lower frequency Alfvén continuum modes are assumed to sit just below the continuum accumulation point, which can be seen in figure 11 at $\rho_{\text {pol }} \approx 0.35$.

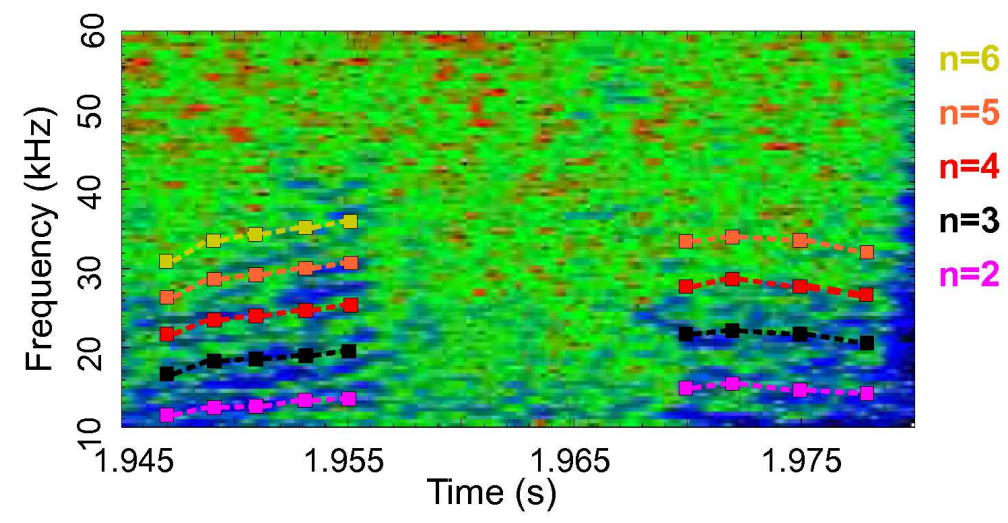

Figure 13. Comparison of numerical results and experimental observations from SXR with tangency radius of $\rho_{\text {pol }} \approx 0.24$ lowfrequency acoustic/Alfvén modes with $n=2-6$ in ASDEX Upgrade discharge 25546.

frequency is found to be of the same order as the low-frequency modes. Due to the low frequency of these modes, they are also much more likely to be influenced by trapped particle effects, with the lower frequency modes being of the order of the ion bounce frequency. Thus, further analysis with full trapped particle effects included in the analytical kinetic dispersion relation [17] are required to fully investigate these modes. The effects of trapped particles on the BAE mode frequency and drive/damping, calculated using the global eigenmode solver LIGKA, has been presented in [7].

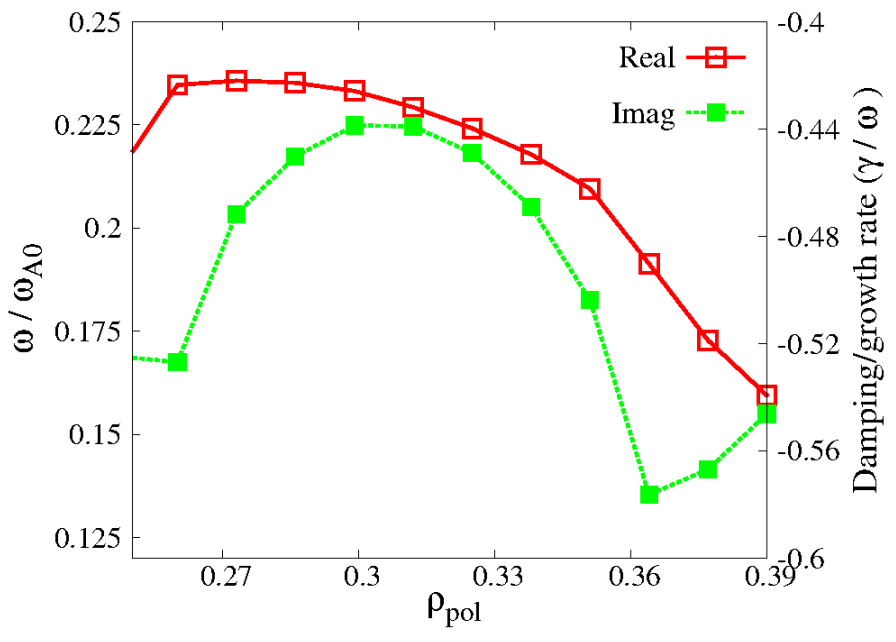

Figure 14. Real continuum and damping rate $(\gamma / \omega)$ for electrostatic drift wave branch for $n=6$ at $t=1.975 \mathrm{~s}$.
It has been presented in [23] that modes, referred to as electrostatic drift waves (e.s. DWs) in the BAAE frequency range, but with much higher damping than the lowfrequency modes presented in the preceding section, are recovered numerically using essentially the same kinetic dispersion relation as equation (1). It is necessary to distinguish between these two continuum branches. Figure 14 shows the $n=6$ real continuum and damping for a third branch recovered by 
solving the kinetic dispersion relation at $t=1.975 \mathrm{~s}$. While the low-frequency modes appear to hover close to marginal stability, this third branch has a frequency close to that of the BAEs in the region close to the $q=1$ surface but is heavily damped. It was found to be difficult to solve numerically due to the very large damping. However it was found to behave as expected with its minimum damping, still up to $\gamma / \omega=-44 \%$, occuring close to the rational surface where it experiences some minimal coupling to the Alfvénic branch. Thus, it is clear that these modes would not be excited experimentally and could not correspond to the low-frequency modes considered in this section. This suggests that this third continuum branch is equivalent to the electrostatic drift wave branch presented in [23], and would account for the large variation in the calculated damping rates between the e.s. DWs and the low-frequency modes presented in this section.

\section{Sweeping modes}

\subsection{Experimental observations of sweeping modes}

During various ASDEX Upgrade discharges, a type of mode that sweeps upwards from the BAE frequency to nearly twice that value has been observed in the final stages of certain sawtooth periods. Discharge 23824, which exhibits mode activity characteristic of BAEs and is a medium density discharges with $I_{p} \approx 800 \mathrm{kA}$, is considered in this analysis. During the time-periods considered, 23824 is heated using 4.66MW ICRH. Figure 15 show mode activity during 23824 measured using SXR channels with tangency radii of $\rho_{\text {pol }} \approx 0.15$ and $\rho_{\text {pol }} \approx 0.35$.

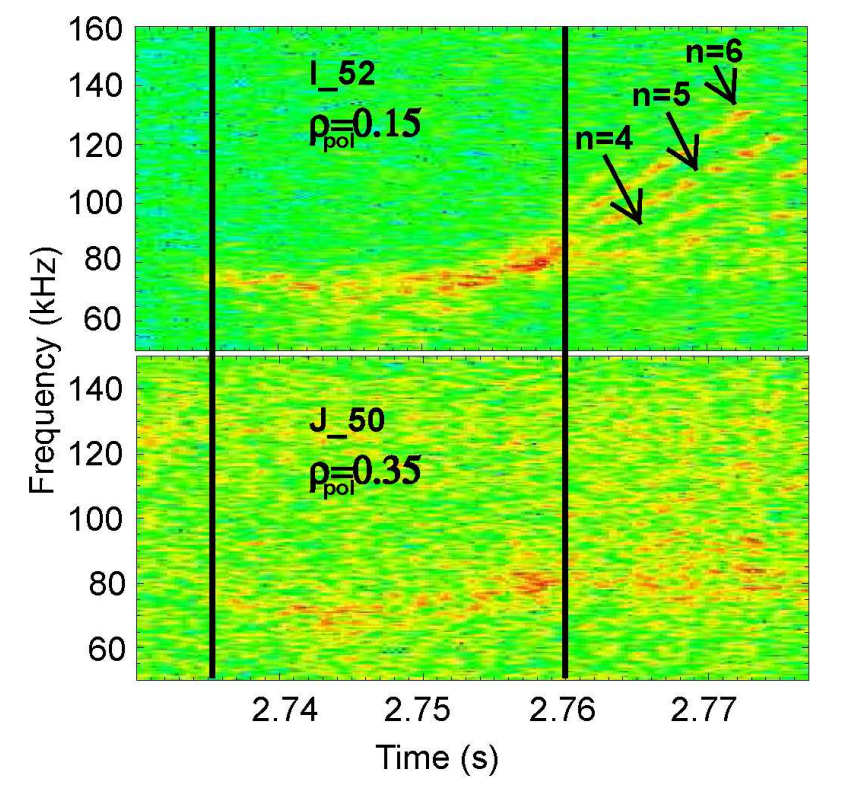

Figure 15. Sweeping mode activity measured with SXR diagnostic during a sawtooth cycle of discharge 23824 at tangency radii of $\rho_{\text {pol }} \approx 0.15$ and 0.35 .
The channel at $\rho_{\text {pol }} \approx 0.35$ continues to show BAE activity from $t=2.735-2.760$ s while the sweeping mode activity from $t=2.760$ to $2.778 \mathrm{~s}$ appears significantly fainter than that observed from the more core localized SXR channel I52. From this it is inferred that the modes observed are of two distinct types - the BAEs with frequencies from $60-80 \mathrm{kHz}$, which are localized in the region surrounding the $q=1$ surface, and the more corelocalized sweeping modes, similar to those observed in [14][34]. Based on the fact that the sweeping modes are clearly visible using edge magnetics measurements from the Mirnov coils, it is taken that they have a strong 
Alfvénic contribution to their polarization. As they are observed in a frequency range well above that of the BAEs and the low-frequency modes where the acoustic contribution to their polarization is expected to be minimal, it is concluded that these modes have a polarization that is predominantly Alfvénic in nature. The splitting and frequency dipping of the BAEs is not as evident as those observed in discharge 25546, but are observed to be caused by the same mechanism. The highest three mode numbers during the sweeping mode phase $(t \approx 2.760-2.778 \mathrm{~s})$ for discharge 23824 , shown in figures 15 and 16, correspond to $n=4,5,6$ based on magnetics measurements using Mirnov coils.

\subsection{Numerical results for sweeping modes}

The $q=1$ surface location from $t=2.730-2.780 \mathrm{~s}$, determined by the same methods described in section 2.3, was found to be $\rho_{\text {pol }} \approx 0.42$. An estimate for the toroidal rotation frequency was obtained from CXR spectroscopy to be $f_{\text {rot }} \approx 3 \mathrm{kHz}$, and each numerically calculated frequency was subject to a Doppler upshift of $n f_{\text {rot }}$.

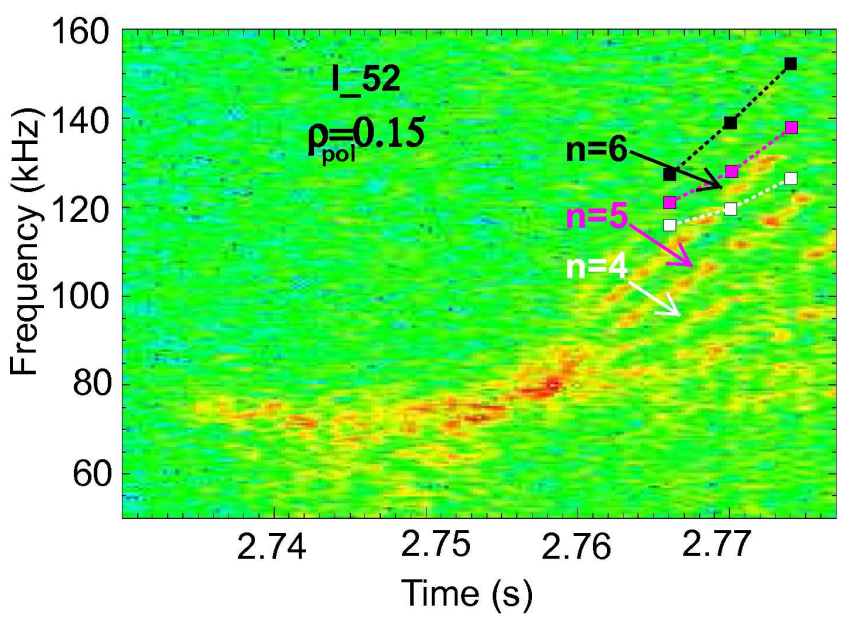

Figure 16. Comparison of numerical results and experimental observations from core SXR channel I52 for sweeping modes with $n=4-6$ during sawtooth cycle of discharge 23824 .

The difference in maximum and minimum $T_{e}$ gradients at the $q=1$ surface is more than two times greater for 25546 than it is for 23824. When this is coupled with the appreciable Doppler upshift in the modes for 23824, the same clear BAE mode frequency splitting is not as evident as for discharge 25546. In fact, no substantial mode frequency splitting is evident in 23824 until $t \approx 2.760 \mathrm{~s}$ when sweeping mode behaviour becomes active, reaching frequencies up to nearly $140 \mathrm{kHz}$ (see Figure 15). Comparing the frequency of the BAE accumulation point close to the $q=1$ surface with the SXR measurements it was found that it can only explain the occurence of the earlier mode activity up to $t=2.760 \mathrm{~s}$ as changes in the temperature gradients are not sufficient to explain such a drastic upward sweep in frequency later in the cycle. Additionaly, in the case of the sweeping modes, the larger mode number splittings are observed to occur at higher frequencies, which is in contrast to the behaviour of the BAEs (see figures 7 and 16). Subsequent sweeping mode activity can be explained by assuming that a new continuum accumulation point is being observed which is more core localized, suggesting that it is caused by changes in the on-axis safety factor value $q_{0}$. This has been investigated previously in [14]. A comparison between the experimental and numerical values for 
sweeping mode frequencies is shown in figure 16, with $q_{0}$ assumed to vary approximately linearly from $q_{0}=0.976$ to 0.962 for $t=2.766-2.774 \mathrm{~s}$. For $n=4-6$ the numerically calculated slopes of the sweeping mode frequencies agree well with experiment with the higher modes having steeper frequency slopes in time. The frequency splitting between the mode numbers is also well reproduced. For example, at $t=2.774 \mathrm{~s}$, the experimentally observed splitting between the $n=4$ and $n=6$ modes is approximately $30 \mathrm{kHz}$ while the numerically calculated splitting is $26 \mathrm{kHz}$. The quantitative agreement is not as close, with differences between $10-20 \%$ in the numerical and experimental results. This could be due to a number of factors, primary among which is the large uncertainty in the core ion density.

\section{Conclusion}

An investigation of low frequency Alfvénic modes observed experimentally during various ASDEX Upgrade discharges has been carried out. Results obtained by solving the low-frequency kinetic dispersion relation $[14,16]$ have been compared with these experimental observations. Various factors, such as the plasma elongation, diamagnetism and realistic safety factor profiles have been included in the analysis. It has been again demonstrated that the kinetic dispersion relation is capable of recovering various low frequency mode branches [7, 14], including those which are of mainly Alfvénic (BAE) and mixed acoustic/Alfvénic polarizations, as well as their respective drive and damping rates. That the frequency evolution of these modes is highly dependant on the diamagnetic frequency $\omega_{* p}$ through background temperature gradients in the plasma has also been demonstrated both experimentally and numerically. Closer agreement is hoped to be found with experiment once an explicit expression for trapped particle effects, which are also dependant on $\omega_{* p}$, is included in a numerical investigation of the kinetic dispersion relation [13]. As the assumption of $T_{i}=T_{e}$ has been made in the calculations for comparison with experiment, the availability of $T_{i}$ profile data may also improve agreement with experiment in future calculations. This has been demonstrated through scans of the evolution of the continuum accumulation point and rates of drive and damping with variations in $\tau=T_{e} / T_{i}$. An investigation into a third branch recovered by the kinetic dispersion relation, the electrostatic drift wave branch, has been undertaken and it has been found to be highly damped. These results have been compared with those from [23]. Further, it has been shown that the on-axis safety factor value $q_{0}$ plays an important role in determining the evolution of low frequency core-localized sweeping modes, which differ somewhat in nature from the BAEs.

\section{Acknowledgments}

The authors would like to thank Dr. Alessandro Biancalani for valuable discussions. This work was supported by the Irish Research Council for Science Engineering and Technology (IRCSET); a research agreement between the Max-Planck-Institut 
für Plasmaphysik, Garching, Germany and University College Cork, Ireland; and EURATOM.

\section{References}

[1] Heidbrink W W 2008 Phys. Plasmas 15055501

[2] Chen L and Hasegawa A 1974 Phys. Fluids 171399

[3] Goedbloed et al 1975 Phys. Fluids 181258

[4] Goedbloed et al 1998 Phys. Plasmas 53143

[5] van der Holst B et al 2000 Phys. Plasmas 74208

[6] Rosenbluth M N and Rutherford P H 1975 Phys. Rev. Letters 341428

[7] Lauber Ph et al 2010 Proc. of the 23rd IAEA Fusion Energy Conference, Daejon, Korea Rep. of (IAEA, Vienna) Vol. IAEA-CN-180 THW/2-2Ra

[8] Cheng C Z and Chance M S 1986 Phys. Fluids 293695

[9] Appert K et al 1982 Plasma Phys. 241147

[10] Sharapov S E et al 2002 Phys. Plasmas 92027

[11] Chu M S et al 1992 Phys. Fluids B 43713

[12] Heidbrink W W et al 1993 Phys. Rev. Letters 71855

[13] Chavdarovski I "Kinetic theory of low frequency Alfvén waves in burning plasmas", Ph.D thesis, University of Tor Vergata (2009)

[14] Lauber Ph et al 2009 Plasma Phys. Control. Fusion 51124009

[15] Mikhailovskii A B et al 1973 Nucl. Fusion 13259

[16] Zonca F et al 1996 Plasma Phys. Control. Fusion 382011

[17] Chavdarovski I and Zonca F 2009 Plasma Phys. Control. Fusion 51115001

[18] Gorelenkov N N et al 2007 Phys. Letters A 370 70-77

[19] Mikhailovskii A B and Sharapov S E 1999 Plasma Phys. Rep. 25838

[20] Gorelenkov N N et al 2009 Phys. Plasmas 16056107

[21] Piovesan P 2008 Nucl. Fusion 48065001

[22] Classen I G J et al 2011 Plasma Phys. Control. Fusion 53124018

[23] Zonca F et al 2010 J. Phys. Conf. Ser. 260012022

[24] Igochine $\mathrm{V}$ et al 2010 IPP Report 1/338

[25] Gao Z et al 2009 Nucl. Fusion 49045014

[26] Mc Carthy P J 2012 Plasma Phys. Control. Fusion 54015010

[27] Mc Carthy P J 1999 Phys. Plasmas 63554

[28] Igochine V et al 2010 Phys. Plasmas 17122506

[29] Rathgeber S K et al 2010 Plasma Phys. Control. Fusion 52095008

[30] Hastie R J 1998 Astrophysics and Space Science 256 177-204

[31] Pietrzyk Z A et al 1999 Nucl. Fusion 18 587-611

[32] Fischer R et al 2010 Fusion Science Technology 582 675-684

[33] Annibaldi S V et al 2007 Plasma Phys. Control. Fusion 49475

[34] Elfimov A G et al 2011 Plasma Phys. Control. Fusion 53025006 